\title{
Research on Design Methods of Interactive Extinguisher Product Based on Usability Theory
}

\author{
Hu Wenfei \\ Wenzhou Vocational \& Technical College, Wenzhou. 325035 \\ 61230934@qq.com
}

Keywords: Innovation; design; extinguisher

\begin{abstract}
Among extinguishers design this time, the fashion style is to cater for the viewpoint and demand of public aesthetics. Meanwhile, the culture and connotation carried by the product is also the necessary factors emphasized by modern urban people. Besides, it also successfully conveys the information of cherishing life to users. In the reality with homogenization of products of the same category in modern times, the enterprise should inevitably manifest unique characteristics by virtue of its product design to occupy the share in market competition. The detail design has direct influence on the overall effect of the design. The essence of product modeling design mainly relies on the product detail. By gradually improving and extracting the product detail and through rational design, it possesses rich detail characteristics. In this way, good foundation could be undoubtedly paid for the design of product image.
\end{abstract}

\section{Introduction}

One of characteristics of the extinguisher is its using method. However, the aiming way of two-hand holding will greatly enhance the accuracy of extinguisher. In the meantime, the burden of single-hand extinguishers in the market on arm could be reduced, so that the females or the weak persons also could use it easily. In this way, the using method of extinguisher is changed. It is indeed a bold try with practical significance and further an important highlight of the product.

To realize modeling design, the designer should actively know about the demands of the user at the present and in the future and pay attentions to the difference at different demand layers and design products could satisfy various demands of different users gradually. The modeling designer should have insight into the development trend of the science and technology and pay attentions to the arrival of new theories, technologies and materials and put them into practice as far as possible, transform advanced technological research achievements into the media of commodities with practical function and also design the products meeting the aesthetic characteristics and cultural inclination of the times. It is inevitable to the designed products according to the conditions of the times, social environment and psychological response. Meanwhile, it is important for the designer to study and predict the tide brought by such change and grasp the design inclination and characteristics.

\section{Scheme selection and description}

The soul of the industrial design is people-oriented. Industrial design indicates a science serving people. Based on such idea, I have changed the using mode of holding the bottle in one hand and nozzle another into that of holding the bottle in both hands and liberating the burden of single hand, and the way of two-hand collaboration enhances the accuracy in operation. In general, it is more humanized and rational. 
Through overall consideration, I decide to select the scheme in the Fig. 1 and 2, and make further improvement on this basis.

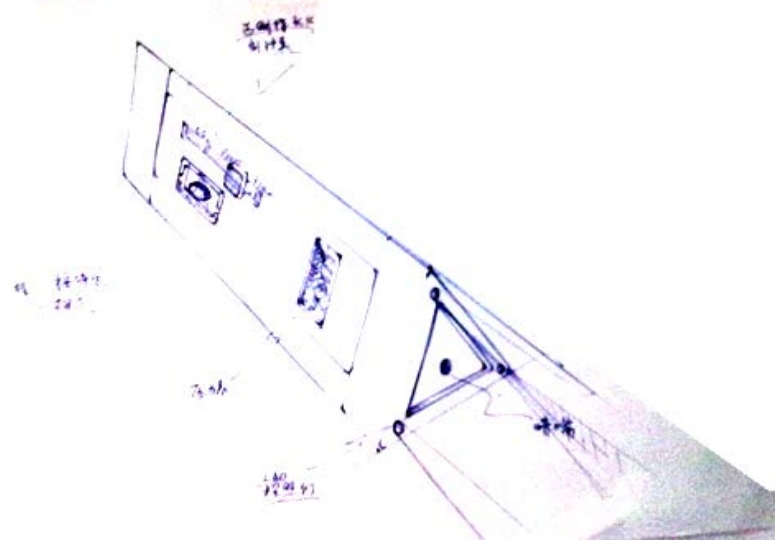

Fig. 1 Design scheme

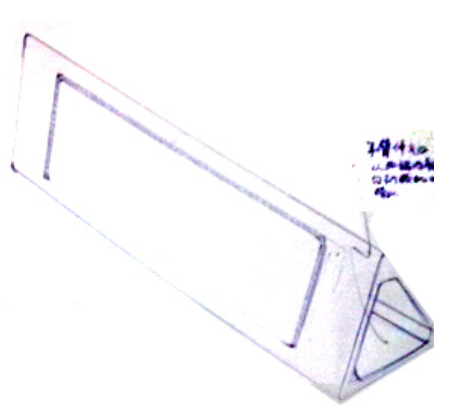

Fig. 2 Design scheme

In final scheme, I change the triangular prism in above Fig. into irregular cylinder to enhance the smoothness and proximity of the product. In this way, the product will no longer be stiff but more applicable.

Fig. 3 indicates the effect of revision. The product inherits the advantages of the designed product over color. The bright and easy-to-recognize red is selected as the main color. In this case, the product can be found out rapidly even under the circumstance of dense smoke to put out the fire immediately.

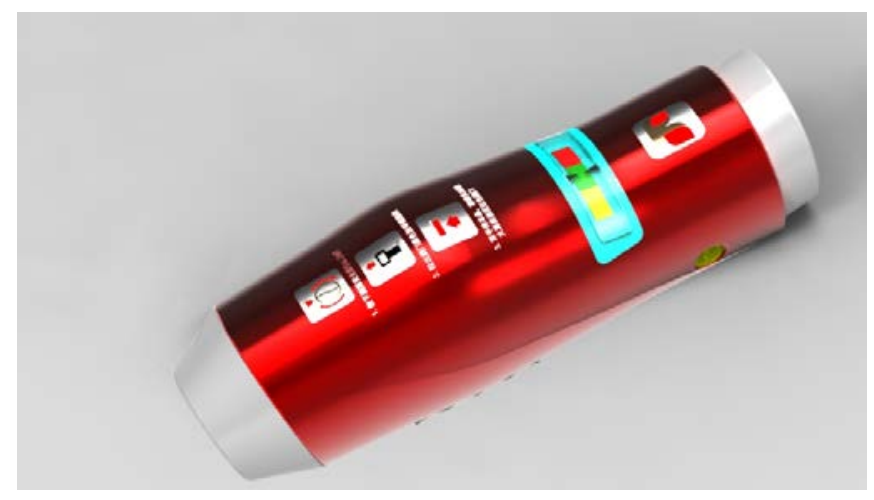

Fig. 3 Effect sketch of revision

Speaking of detail design, the pressure gauge of the product is improved so that the pressure of extinguisher can be easily read no matter when it is used or when it is placed. Thus, it is convenient in using and overhauling. The reading standard is in strict accordance with national related regulations: it indicates under pressure when the pointer points to red, then it can be used unless a little pressurized; it is normal when it points to green. Meanwhile, it is high pressure when it points to yellow.

Speaking of the safety design, children's safety knob is added to the product namely the yellow safety knob in the picture below. When using the product, the user needs to press the knob and rotate it to the proper location, then the it can be used after the safety pin is pulled away, or the product will not spray even if the user pulls away safety pin and presses the valve. It is necessary nowadays when the importance is attached to the children's safety.

\section{Color scheme}

After determining the scheme, I tried a lot of color schemes including blue that clams one, black that is stable and restrained and yellow that is bright and dynamic. However, with consideration given into the fact that the actual application environment of the extinguisher is related to the state, I finally choose the easy-to-recognize red as the main color, and use little white that is concise and 
has strong sense of technology. Yellow is embellished in some important parts for highlight.

Foreign research and practice shows that the success in product detail design usually can bring market success to the enterprise and lay good foundation for shaping product image of the enterprise so that the purpose of improving the market competitiveness can be reached. Developed countries including America, Japan, German and France have entered post-industrial age in succession. Along with the development of science and technology, the design idea has been gradually improved. As for the essence of product modeling design, it mainly depends on the product detailing which shows the understanding about culture. However, the design's proficiency can further be revealed through detail treatment. Product detail design is an important standard for design evaluation for the sense of beauty is hidden in detail characteristic. Young users have thirst for beauty and are particular about it in daily life. When using the products, they are willing to find and appreciate the exquisite product detailing. Along with the detail treatment, the extinguisher can be easily distinguished from those in the market, so that the product is firmly kept in mind by users.

\section{Design sketch scheme}

The scheme is as shown in the Fig. 4-7.

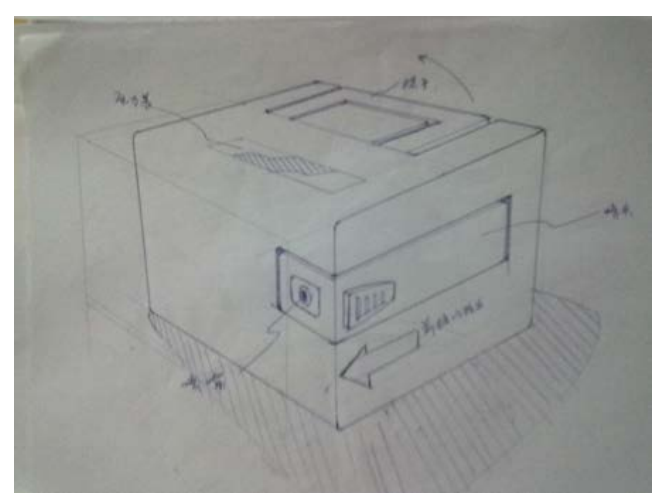

Fig. 4 Sketch

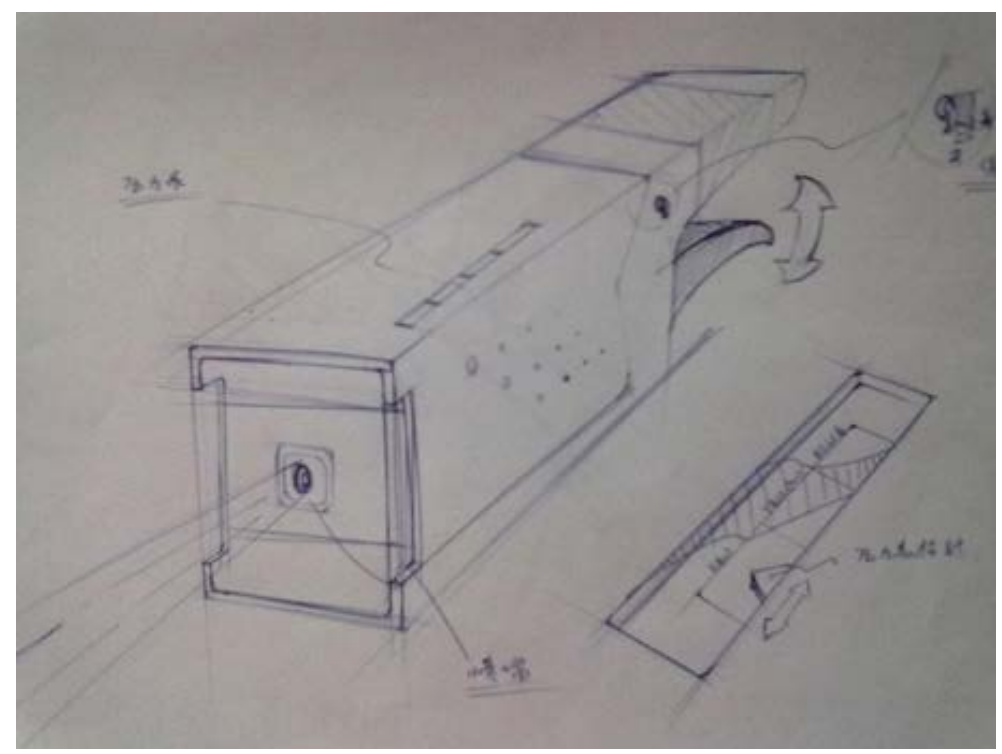

Fig. 5 Sketch 


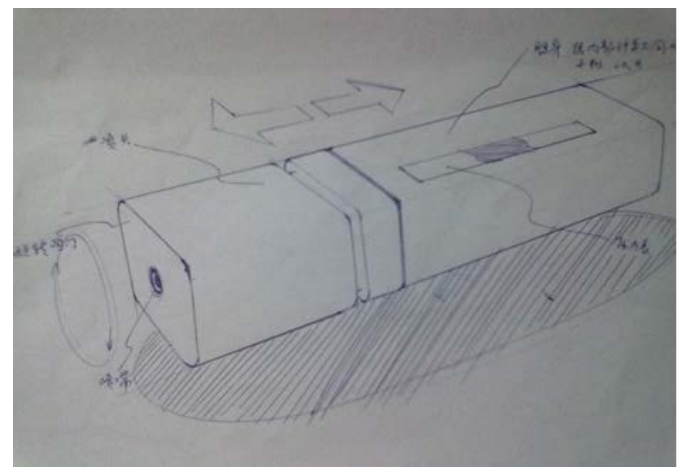

Fig. 6 Sketch

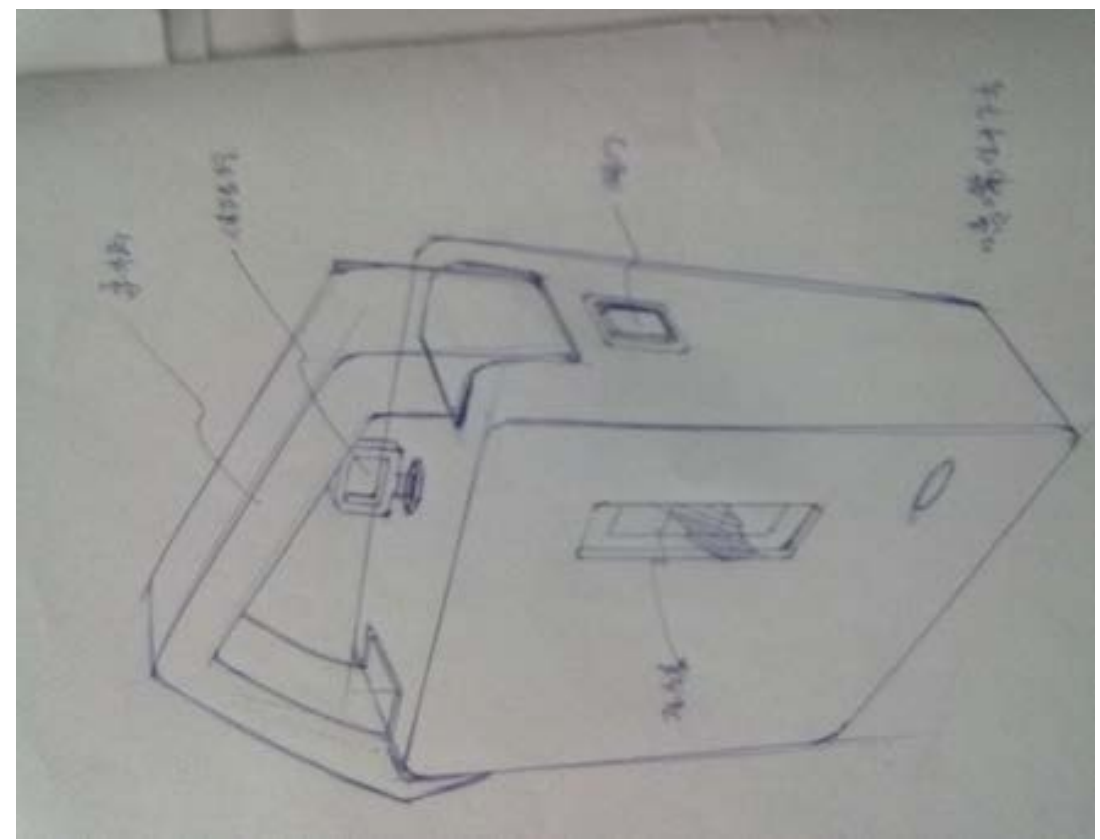

Fig. 7 Sketch

\section{CAD design}

\subsection{D modeling}

Fig. 8 is $3 \mathrm{D}$ view during design.

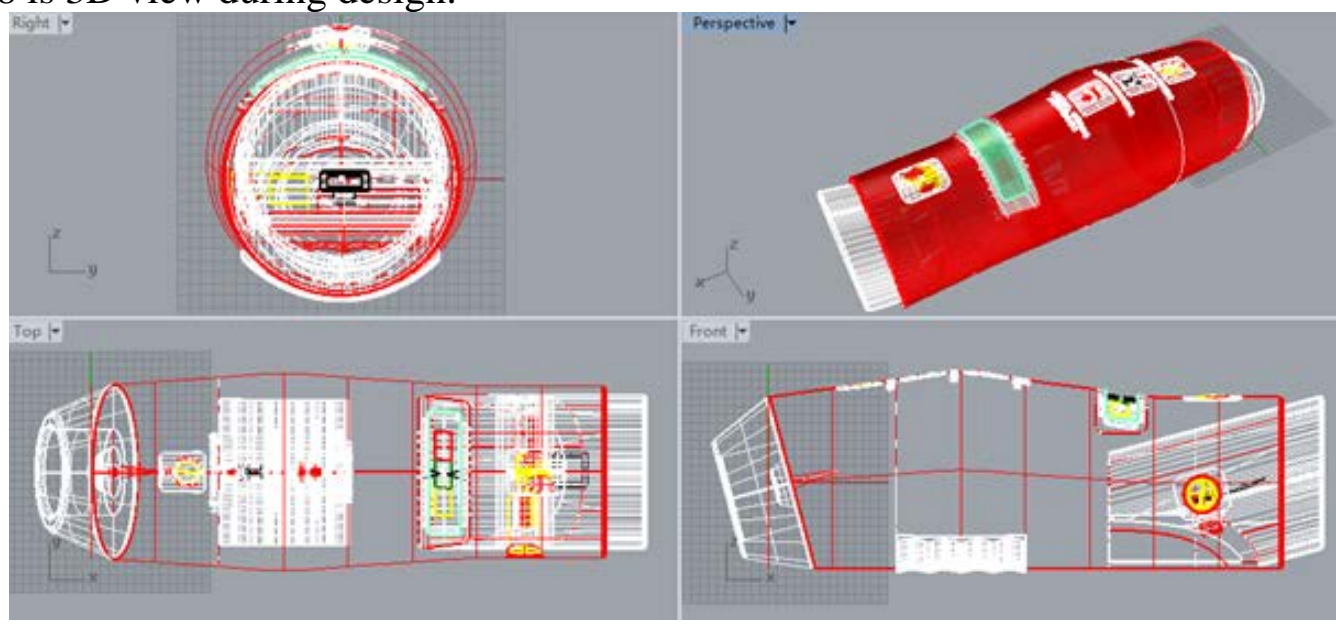

Fig. 8 3D view 


\subsection{Model rendering}

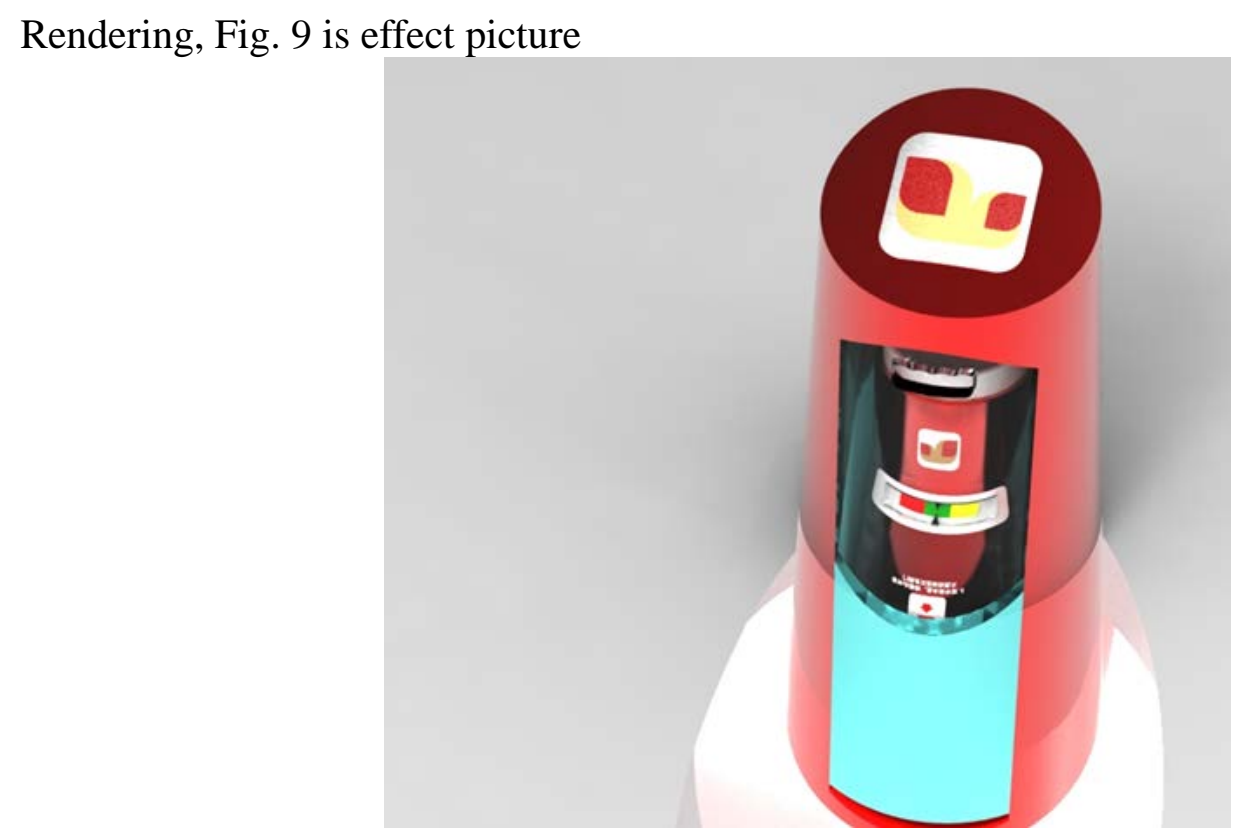

Fig. 9 Extinguisher and storage box

The so-called industrial design indicates a burgeoning interdisciplinary involving technology and art finally formed in modern industrial period. As a theory and method of modern design, its research content not only includes product function, structure, material, manufacturing process and morphology, color, surface treatment and decoration craft of the product but also includes many factors related to the society, economy, people's physiology and psychology. Through basic theories and measures of modern design, modern industrial products may bring about the feeling of efficiency, comfort and beautiful appearance to the users as far as possible to fully satisfy people's material and spiritual demands.

\section{Product material}

As abovementioned, design is a comprehensive project. A lot of factors should be taken into account. Especially during actual production, we cannot simply think that the sense of which material is the best and most environmentally friendly. With many categories of design materials and wide scope, it is a practical and important problem to correctly and rationally choose materials in design. The selection of design materials should follow the following principles:

Appearance: the consideration should be given into the feeling characteristic of the material. It is necessary to choose the material with the best texture and most environmentally friendly according to the modeling, times and purpose characteristics of the product.

Inherent characteristic: the inherent characteristics of the materials shall satisfy the demands of the product function, using environment, operation condition and environmental protection.

Technology: the material should possess good technological performance, meets requirements of the modeling technology, processing technology and surface treatment and shall be adapted to the processing equipment and production technology.

Production cost and environmental factor: based on satisfying the design requirements, reduce the cost, choose the materials with rich resource, cheap price and favorable for ecological environmental protection as far as possible.

Innovation: the arrival of new material provides wider premise of the product design and satisfies the requirements of product design.

In addition, market economy shall follow economic principle. That is, the optimal result can be achieved at the lowest cost. The designer also should follow this principle to reduce design expense and make excellent products. Of course, cheap price should be blindly pursued for rough 
manufacturing. In this case, the fundamental principle of the product design will be violated and the product is also not competitive in the market. For this regard, we should know well about the performance, production modes and ways of various materials and try to reduce the cost under the premise of not damaging the performance and modeling sense of beauty.

There is variety of textures, the material of the handler is stainless steel and plastics and that of valve body is copper and the surface should be passivized, or chrome plate or nickel treatment can be made. The material of the nozzle is the plastics and part of it is ABC plastics, surface electroplating; metal nozzle is mostly made of copper. The bottle body is made of steel, in which carbon dioxide extinguisher is seamless steel bottle.

\section{Conclusions}

Industrial design is different from the traditional engineering design for it gives full considerations into the improvement of the structural performance index of the product and various factors related to the product, society, market, people's physiology and psychology. It is also different from general art design for it lays emphasis on morphology art of modern industrial products and the economic value that morphology and function of the product should be unified with its morphology and production. Therefore, industrial modeling design refers to the creative activity of organically unified science \& technology, fine arts and market economy. In general, whether industrial design is fine arts or not but the road of beauty.

\section{References}

[1]Ki-Young Jeong,Jae-Dong Hong. A simulation study for fire extinguisher manufacturing process layout design[J]. International Journal of Management Science and Engineering Management,2009,4(4).

[2]Julian F.V. Vincent. Biomimetics in architectural design[J]. Intelligent Buildings International,2016,8(2).

[3]Zhuo, Jiang,Yinshui, Liu,Xufeng, Zhao,Wudi, Fang. A portable piston-type water mist fire extinguisher for spacecraft[J]. Proceedings of the Institution of Mechanical Engineers,2015,229(1).

[4]Burnett, Brandon. Simplifying the Hose Stretch with Strategically Placed Loops[J]. Fire Engineering,2015,168(10).

[5]Anonymous. Portable mini crane[J]. Concrete Products,2016,119(6).

[6]Wahyu Sapto Aji,Fajar Hermawanto,Muchlas. AT89S52 microcontroller Based A Fire Extinguisher Robot Using Ultrasonic and Ultraviolet Sensor[J]. TELKOMNIKA,2009,07(3).

[7]Crumière P P,Albaladejo R,De Saint Maurice G,Trabold F,Mc Nelis U,Boileau S,Bouaziz H. [Patient associated fire in theatre: Are we ready to prevent and/or manage it? Assessment of the anaesthetist's knowledge in Lorraine.][J]. Annales Francaises d'Anesthesie et de Reanimation,2012.

[8]Cheng-Chan Shih,Richard S. Horng,Shin-Ku Lee,Egidijus R. Vaidogas. Investigation of Lab Fire Prevention Management System of Combining Root Cause Analysis and Analytic Hierarchy Process with Event Tree Analysis[J]. Mathematical Problems in Engineering,2016,2016.

[9]Tammy Eger,Alan Salmoni,Robert Whissell. Factors influencing load-haul-dump operator line of sight in underground mining[J]. Applied Ergonomics,2003,35(2).

[10]Eger Tammy,Salmoni Alan,Whissell Robert. Factors influencing load-haul-dump operator line of sight in underground mining.[J]. Applied Ergonomics,2004,35(2). 